\title{
Thinking on the Teaching of Integral Theory in Higher Mathematics
}

\author{
Yiyuan Shao ${ }^{1}$, Wenbin Liu ${ }^{2, *}$ \\ ${ }^{1}$ School of computing,Ezhou University,Ezhou , P.R. China, 436000 \\ ${ }^{2}$ Wuhan Technology and Business University,Wuhan ,P.R. China,430065
}

Keywords: Integral theory; Application ability; Integral thought;Calculus teaching

\begin{abstract}
Through the summary and analysis of the present calculus teaching, it is pointed out that the teaching of integral theory should not be confined to the concept of paying attention to knowledge and skill in the traditional teaching materials, but should focus on digging its profound ideological connotation and application ability. Through the knowledge of mathematical history of calculus and the relevant realistic background, this paper permeates integral thought by analyzing the relationship of the indefinite integral, the definite integral and the differential, and puts forward some countermeasures and suggestions to improve the formalized ability of Mathematics.
\end{abstract}

\section{Introduction}

At present, the level of higher education in China has undergone tremendous changes, and the corresponding educational ideas and teaching contents must also be adjusted and optimized [1]. In the calculus teaching of higher mathematics, mathematics teachers should give students two things. The first is the idea of mathematics, which is to let students learn to think and solve problems by using the thought method of Mathematics. And then it's applied ability (which is especially important for non-math majors), which is to let students learn to use mathematics to find out the problems and to sum up, to cultivate the students' mathematical formalization ability, to mathematically transform the actual problems, and to further formalize the mathematical algorithms. In the current calculus teaching, students always find the following three blind spots: (1) It is difficult to understand the relation between definite integral and indefinite integral concept from definition. (2) The reciprocal relation between differential and integral is ambiguous. (3) There are obstacles to the connection between practical problems and mathematical knowledge in the application process. Therefore, the practical mathematical theory, such as calculus, has not been grasped by the students, thus unable to improve their application ability.

\section{Unreasonable factors in traditional integral theory teaching}

The reasons for the above blind spots are mainly due to the obvious deficiency of traditional calculus teaching (including teaching materials).

(1) It does not conform to the cognitive law and historical development. In theory, differential and indefinite integrals are the concepts after definite integrals [2], the arrangement of traditional teaching materials is different from the real process of theoretical development. First of all, the students have to do a bunch of puzzling antiderivatives and then tell them why they're doing it, Although there is no logical problem in this arrangement, and the operation is very systematic, but it affects the understanding of the concept of definite integral. Historically area problem is very old, and an important background of definite integral is to solve area problem. It is only after the 
newton-leibniz formula is produced that the area problem is easy to solve. The real process of historical development in teaching is conducive to the mastery of integral thought.

\section{Measures to improve unreasonable factors in traditional integral theory teaching}

What is the best way to deal with the integral theory in the current textbooks? I personally think that, compared to the traditional integral teaching, it may be necessary to find a teaching method that is more consistent with the cognitive law, the students are easy to accept and better run through the mathematical ideas. Calculus is the most practical mathematical theory, which is related to its production in natural science. Therefore, in the course of teaching, we should not leave this big background, avoid calculus as pure knowledge and skills to teach. Students' understanding of calculus should not only stay on books. On the basis of traditional textbooks, more attention is paid to the mastery of the method of integration and the training of the ability to use.

Make some minor adjustments in the order of integral contents of the current teaching materials, a review of history shows the significance of the study of integrals. After studying the differential theory, first, why do we need to study integrals? We can start by looking at historical facts. Whether in ancient China, Liu Hui's "cutting circle" or "Exhaustion method " of Archimedes in ancient Greece, Area is a very difficult problem, and solving this problem often requires great skill. It is permeated with the basic ideological prototype of "replacing curve by straight line" and the main idea of the calculus of "infinite partition, infinite sum". Another example, whether it is the principle that the Chinese ZuShi father and son 's "with both power potential, product intolerance", or the Italy mathematician Cavalieri's "non component" volume method is the pioneer of integral calculus, which is related to the current definite integral and double integral definition and the microelement method. Let students understand the core of calculus is replacing complexity with simple approximation locally, and then reach the accuracy through the limit, emphasizing from the derivative problem to the integral problem all reflect this idea.

Introduce the concept of definite integral in accordance with the research background of calculus. Through reviewing history, the origin of calculus mainly came from the need to solve two problems: mechanics and geometry. That is to introduce the concept of definite integral with the traditional typical example road and area problem, and then explain the difficulty of calculating the definite integral by the definition of definite integral. However, it is shown from the road model that the definite integral of the speed function $\mathrm{V}(\mathrm{t})$ on the interval $[\mathrm{a}, \mathrm{b}]$ equals the increment of the range function $\mathrm{S}(\mathrm{t})$ on the interval [a,b]. The values of $\int_{a}^{b} d x$ and $\int_{a}^{b} x d x$ that are calculated by the geometric meaning of the definite integral. According to the geometric meaning of the definite integral of these two functions, there are : $\int_{a}^{b} d x=b-a, \int_{a}^{b} x d x=\frac{1}{2}(a+b)(b-a)=\frac{1}{2} b^{2}-\frac{1}{2} a^{2}$, that is, the definite integral of function $f(x)=1$ and $f(x)=x$ on the interval [a,b] equals the increment of function $f(x)=x$ and $f(x)=\frac{1}{2} x^{2}$ on the interval [a,b].

And we know that the original function of the velocity function $\mathrm{V}(\mathrm{t})$ is the distance function $\mathrm{S}(\mathrm{t})$, and $x^{\prime}=1,\left(\frac{1}{2} x^{2}\right)^{\prime}=x$. Thus, it is judged by intuitive experience that calculating the definite integral is of great importance to the original function of the integral function, and puts forward the necessity and reality of discussing the original function.

Dilute skill training and use mathematical software to solve indefinite integral. Based on the 
previous study of definite integral concept, the indefinite integral is introduced to emphasize the background of the actual problem of indefinite integral: For example, the velocity $v=v(t)$ of particle for the linear motion of the variable speed is known to seek the road function $s=s(t)$. The relation between the slope of the tangent line and the abscissa is known, and the equation of the curve is obtained. The marginal cost function of a commodity is known to find the total cost function. These problems are a unified mathematical model: For a given function $f(x)$, find derivable functions $F(x)$, and make $\quad F^{\prime}(x)=f(x)$. Thus we define the concept of original function (inverse derivative) and indefinite integral. Although the operation of indefinite integral has many skills, the general indefinite integral technique can not form a certain method. The meaning of integration by substitution and integration by parts is only to change the form of the problem, but in fact it can not be called "method". The change of form may develop in the direction of easy solution, or it may be more difficult to solve. It is not necessary for students of non mathematics major to expend too much energy. On the one hand, the integral table can be used to check the desired results in the table after the integral function is directly or simply deformed. On the other hand, many mathematical software can easily calculate indefinite integral, such as Maple, Mathematica, etc. It can be introduced in a small number of examples in class. In addition, the approximate calculation of the definite integral by the computer can also be introduced properly. It does not take much energy. The definition of the definite integral itself suggests how to program.

Using the geometric meaning of definite integral to determine the relation between integral and indefinite integral. The current higher mathematics teaching of the newton-leibniz formula is generally adopted: First, the integral upper limit function is defined, and a primitive function of $\mathrm{f}(\mathrm{x})$ is given in the form of definite integral with variable upper limit, and then the formula of Newton-Leibniz is proved. This teaching method is rigorous and complete in theory, and pays attention to the logical structure of the content. However, the teaching process is abstract and boring, ignoring the psychological characteristics of the students, and is not conducive to the exploration of the relationship between the definite integral and the indefinite integral.

Let $\mathrm{f}(\mathrm{x})$ be continuous on $[\mathrm{a}, \mathrm{b}]$, then $\mathrm{f}(\mathrm{x})$ has primitive function on [a,b], and guide students to find the relationship among $\mathrm{f}(\mathrm{x})$, definite integral and original function. Because the background of the definite integral concept is based on the area, it is related to the geometric meaning of the definite integral, and the structure of its area is discussed.

Using $\mathrm{f}(\mathrm{x})$ and the geometric meaning of definite integral to construct "area function" $\mathrm{A}(\mathrm{x})$, it is possible to assume $\mathrm{f}(\mathrm{x})>0$. Take an arbitrary pointx within [a, b], Using $\mathrm{A}(\mathrm{x})$ to represent the area function surrounded by $X$ axis, $x=a, x=x$ and $y=f(x)$. Obviously, $A(x)$ is a continuous function of $x$ $\in[\mathrm{a}, \mathrm{b}]$.

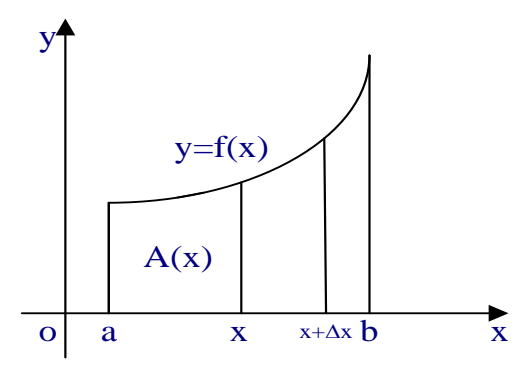

Figure1. Illustration of "area function" A (x) 
As shown in the Fig. 1, there is:

$$
f_{\min } \Delta x \leq \mathrm{A}(x+\Delta x)-\mathrm{A}(x) \leq f_{\max } \Delta x(1)
$$

When $\Delta x \rightarrow 0$, there are $f_{\min } \Delta x \rightarrow 0$ and $f_{\max } \Delta x \rightarrow 0$, and so:

$$
\begin{array}{r}
\mathrm{A}(x+\Delta x)-\mathrm{A}(x) \rightarrow 0 \\
f_{\text {min }} \leq \frac{\mathrm{A}(x+\Delta x)-\mathrm{A}(x)}{\Delta x} \leq f_{\text {max }}(
\end{array}
$$

Because $\mathrm{y}=\mathrm{f}(\mathrm{x})$ is a continuous function, when $\Delta x \rightarrow 0$, both $f_{\min }$ and $f_{\max }$ tended to be the same value $\mathrm{f}(\mathrm{x})$. Therefore:

$$
\lim _{\Delta x \rightarrow 0} \frac{A(x+\Delta x)-A(x)}{\Delta x}=\mathrm{f}(\mathrm{x})(4)
$$

That is:

$$
A^{\prime}(x)=\mathrm{f}(\mathrm{x})(5)
$$

This shows that $A(x)$ is a primitive function of $f(x)$, that is, we call $A(x)$ the upper limit function of integration, which is defined as $\mathrm{A}(\mathrm{x})=\int_{a}^{x} f(t) d t$.

By using the area function constructed by the geometric meaning of the definite integral, the structural origin of the integral upper limit function is explored,and the upper limit function of integral is no longer the theoretical knowledge of abstract boring and artificial construction, and the method of combination of number and graphics embodies the visualization and vividness of the integral upper limit function. From this, the result is obtained that if $\mathrm{f}(\mathrm{x})$ is continuous on [a,b], then the indefinite integral of $\mathrm{f}(\mathrm{x})$ on $[\mathrm{a}, \mathrm{b}]$ can be expressed as definite integral.

$$
\int f(x) d x=\int_{a}^{x} f(t) d t+\mathrm{C}+\mathrm{C}(6)
$$

It reveals the intrinsic and essential relation between the indefinite integral and the definite integral, and also reveals the relation between the derivative and the definite integral, and the derivation operation is the reciprocal operation of the variable upper integral calculus.

Deepen the understanding of relations between differential and integral from the perspective of mathematical philosophy. The idea of calculus makes dialectics infiltrate into mathematics, and it also makes mathematics a powerful tool to accurately express the laws of natural science and technology and to solve problems effectively. In teaching, strengthening the mathematical culture in the variable mathematics, such as the relationship between "finite and infinite", is a philosophical problem and a mathematical problem. In the theory of calculus, the finite problem, that is, "the total amount of non uniform distribution" (the area of the trapezoid, the distance of the linear motion of the variable speed, the profit of the price with the change of sales) is indefinitely divided, and the "uniform distribution" (the rectangular area, the distance of the uniform linear motion and the profit of the Pricing Marketing) is obtained, then they are also asked to obtain infinite sum and obtain the process of limited problems (the area of trapezoid shape, the distance traveled, and the marketing revenue). That is, the infinite process is used to deal with the finite problem. The process of infinite change is only a powerful mathematical method - the limit, ingeniously through the analysis of the 
"trend" of the finite situation, and the ultimate value of an infinite process is obtained. It also permeates another kind of opposite relationship "whole and part". The process of infinite segmentation can make the whole (curve, variable speed, variable price) become local (straight line, uniform speed, pricing), and then Infinite summation makes the local (straight line, uniform speed, pricing) into a whole (curve, variable speed, variable price). The most elaborate embodiment: the infinite segmentation from the whole to the part is a dynamic process; on the contrary, the infinite summation from the local to the whole are also the process of deepening the whole. Only under the condition of infinite division they realize the mutual transformation, from the opposition to the unity, which embodies the thought of " breaking up the whole into parts, the approximate replacement, accumulation of zero as a whole and infinite approximation" of the definite integral.

\section{Summary}

Integral is widely used in various branches of natural science and social science. It is involved in the teaching materials, including geometric, physical, and economic and so on. However, no matter which kind of problems can not be separated from the thought of integration. Only in the process of teaching running through that the definite integral is a kind of tool for people to solve practical problems by using mathematical ideas, and the actual problem can be abstracted as a mathematical form of definite integral.

\section{Acknowledgement}

This work was supported by science and technology research project of educational commission of Hubei Province in China (B2016538). The authors would like to thank all the participants in the experiments.

\section{References}

[1] Department of Applied Mathematics of Tongji University, Advanced mathematics, Higher Education Press, Beijing, 2002.

[2] LI Gaiyang, LUO Debin, Wu Jie etc, Appreciation of mathematics culture,Science Press, Beijing, 2011.

[3] HUANG Limin,HUANG Nanchen, Adjustment and reform of infinitesimal calculus content in calculus course, J. STUDIES IN COLLEGE MATHEMATICS. 11(6)(2008)28-30.

[4] LI Decai, LUO Rujiu,ZHANG Wenjun, Stratified mathematics, Jiaotong University press, Beijing ,2007.

[5] D. Varberg, E.J.Putcell, S.E.Rigdon, Calculus, Prentice Hall, Lundun,2006, 